\title{
Architectural Considerations for Personal Mobility In the Wireless Internet
}

\author{
Mazen Malek Shiaa and Finn Arve Aagesen \\ Department of Telematics, NTNU University, Trondheim - NORWAY \\ \{malek,finnarve\}@item.ntnu.no
}

\begin{abstract}
Personal Mobility, and the need for it, is considered to be the main driving force behind the spreading of the Wireless Internet. Moreover wireless devices and applications are being developed based on a basic assumption that users need to get access to services regardless of their location and used equipment. Wireless Services, on the other hand, are very dynamic and have a vibrant configuration and settings. In order for this to be utilizable a new network architecture is required. In our department we are developing Telecommunication Architecture for Plug-And-play Systems (TAPAS) to be a generic platform and application development environment based on plug-andplay technology, which uses the Web as means for service definition, update and discovery. TAPAS is intended to satisfy three basic classes of properties: Flexible and adaptable, Robust and survivable, and QoS aware. Personal mobility falls into the first class. Aspects of user, application and session mobility are dealt with using different mobility management schemes. The paper discusses trends in the Wireless Internet concerning devices, applications and services, and presents the approach used within TAPAS. Personal mobility in this context is based on the user, terminal and session mobility.
\end{abstract}

Key words: Personal Mobility, User, Device, Application, Session, Plug and Play

\section{TRENDS IN THE WIRELESS (INTER)NET: DEVICES AND APPLICATIONS}

Wireless Internet is the network of radio-connected user devices and servers using various services. Different players, such as governments, standards bodies, manufacturers, and commercial enterprises, are preparing

The original version of this chapter was revised: The copyright line was incorrect. This has been corrected. The Erratum to this chapter is available at DOI: 10.1007/978-0-387-35618-1_37 
the telecommunication industry for an upgrade of cellular phone services for a wireless Internet. The PC industry, is stepping up pace for providing the mobile users with wireless access through usual desktop environment.

To provide some aspects of the "Big Picture" one should answer the following question: which Platform is suitable for Wireless Internet services, and which devices and technologies are available for it?

There are different kinds of wireless devices capable of participating in wireless Internet services:

- Java phones: Normal cellular phones with the ability to run Java.

- Wireless PDAs: Wireless handheld devices.

- Communicating appliances: e.g. cameras, wireless printers, barcode scanners, pens, etc.

- Wireless PC: Laptops and notebooks with full desktop capabilities.

All of these devices fall into one of two main categories: Tiny or PC-like computational power. This concerns the onboard processor, memory, display, control, etc. These devices are available on limited scale, however their characteristics need to be improved to run content-rich applications.

In terms of services a variety is available for specialized persons and markets. These services use different mark-up languages to achieve poor content services, mainly browsing, such as WML (for WAP users), cHTML (for i-mode phones), HDML (for handheld), etc.

The key applications of any wireless system will be of complex interactive nature with rich and dynamic content, which cannot be fulfilled by simple browsing means. Applications in Commerce (mobile transactions with high security), Transportation (search for complex airline information, reservations), Airports (queue free check-in), customer relationship (automatic upgrades, remote service checks), etc. could all benefit from a Plug and Play platform that has full support for various mobility scenarios and built on common programming interface.

\section{UNDERSTANDING PERSONAL MOBILITY}

To see what will be future's networks and services, understanding the nature of people is needed. Users are mobile and what they keep on their person is important. A mobile person drives personal content, wireless applications, and even mobile hardware. Therefore, Personal Mobility is defined by: " $a$ utilization of services that are personalized with end user's preferences and identities independently of both physical location and specific equipment."

A model, thus, is needed to represent the mobile user with all its profile and preferences, applications and sessions, devices, location and access 
point, underlying operating system and domain policies, etc. There are certain factors that play crucial role in defining the overall structure and complexity of any given model or platform. User-to-device relation could be utilized in different ways; e.g. exclusively one user per device, different users controlled by SIM cards, different users determined at login phase, etc. Another factor could be the mobility of Personal content, which is determined by the flexibility of moving user's specific information and preferences to the user's location and device.

\section{TOWARDS A NEW NETWORK ARCHITECTURE}

Addressing Personal mobility in a dynamic environment in which both resources and services vary in terms of availability and configuration is a real challenge. As introduced in the previous sections, figuring out what is mobile is half of the problem, keep it functioning is the other half. A vital and initial requirement is the availability of the services, or the loading of the application. When services are subject to dynamic change regarding their functionality, e.g. different versions at different domains, the platform should provide the means for automatic reconfiguration and update at every node and terminal. Once a terminal is accessing a service it should be able to keep on accessing it transparently of any further network and application management tasks. Issues such as resource (re)allocation, service (re)adaptation and dynamic (re)configuration should also be dealt with.

Requirements of platform to exploit the full power of mobility in the Wireless Internet could be:

- To be Person based and not device based.

- "Anytime and anywhere" installation and operation.

- Freedom for content providers and support for dynamic content.

- Automatic discovery of new components and their services.

- Support for different capabilities of devices.

- Continuous adaptation to the environment.

There are additional requirements that are implementation dependent and depend on the type of development environment and the applications:

- Expressiveness: what interactive methods possible at user device

- Availability: How to apply different addressing schemes (physical and mobile) for different devices and applications.

- Reliability: What central components to be replicated.

- Personality: How to define User session and User profile. 


\section{TAPAS AND ITS APPROACH FOR PERSONAL MOBILITY}

\subsection{What is TAPAS}

TAPAS is a generic platform and application development environment that uses plug-and-play technology to provide flexible means for network operational tasks such as installation, configuration and management [1]. The basic model is founded on the concept of the Theatre metaphor, where actors perform according to a predefined manuscript and a director manages their performance. Actors could be software components running in a wireless device providing the user with certain functionality. Once functionality plugged in, it is possible to configure it, adapt it to the environment, manage it and move it. Figure 1 shows how different objects are related to each other in the TAPAS terminology. It is based on the director-actor relation, as well as the node-domain relation. Services and applications (or ServiceSystems) are stored as plays (different manuscripts for different Roles), while executed and performed by ApplicationActors. Dynamic control is provided through the CapabilitySupport that handles all resources and capabilities offered by nodes and domains, e.g. transmission channels and IP addresses. The Plug-in phase of every ApplicationActor, on the other hand, defines the quality at which it is going to execute.

Node mobility is supported through dedicating special purpose actors at each device, Mobility Agents, which are controlled by the Mobility Manager. There are two basic schemes for this kind of mobility management; device location update and node discovery [2]. This paper focuses on the Personal Mobility issues and support provided to applications.

\subsection{Representation of Personal Mobility in TAPAS}

What is central to our approach is the terminal identification and mobility of functionality. Within the TAPAS framework terms and notions on Personal mobility have been defined and related to active entities in the system. For example:

- person/persona is referred to by an ID and profile,

- user-to-device relation is defined at login phase,

- a user-application interaction is controlled by a UserAgent,

- every time a user logs in a user session is maintained,

- personal content is defined by applications and is downloadable,

- applications are dependable on complex capability system regarding device, domain and environment characteristics. 


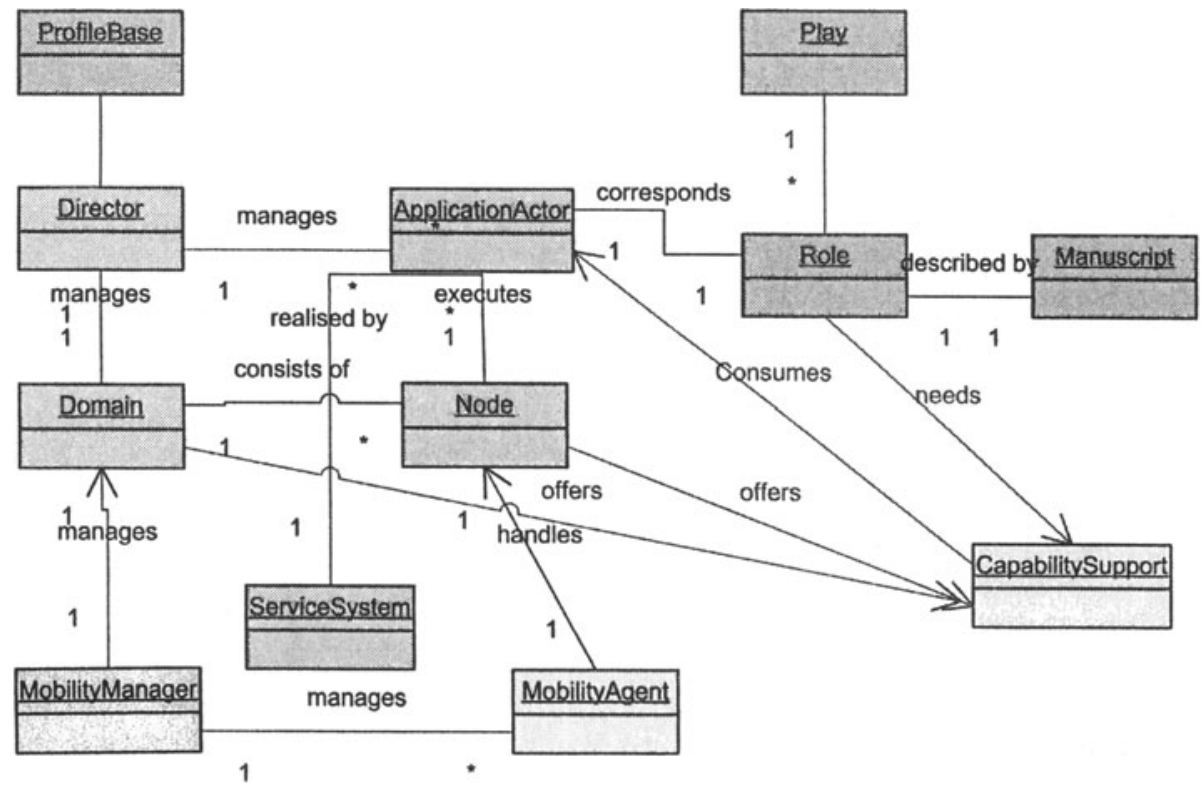

Figure 1. The object model of TAPAS.

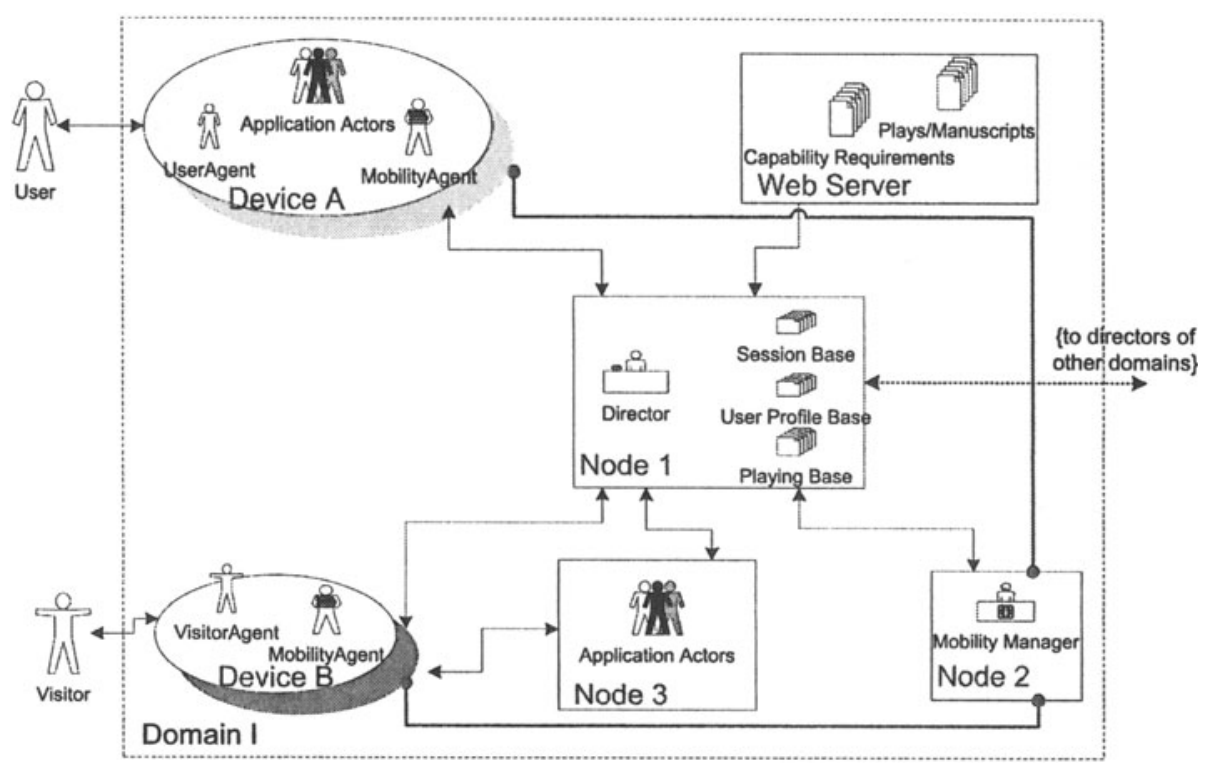

Figure 2. Terms used for Personal Mobility in TAPAS. 
Figure 2 provides a general overview of how Personal Mobility is handled by TAPAS. The devices depicted are characterized by different set of capabilities, that's why certain application components run at networks nodes instead of user's device. For purpose of multi-domain environments the domain's director, as there is only one director per domain, could contact other directors inquiring about visiting user's ID and profile.

\subsection{User and Session Mobility}

In TAPAS user's interactions are controlled by UserAgent and user sessions are maintained by the director in terms of Session descriptions, which is a detailed sketch of running applications, actors and their related data. Figure 3 demonstrates how user's session is managed by the UserAgent, and consequently maintained by the director's data base. An example is provided for a session description and a user profile. In this example application actors are distributed on the user's device and a network node, a typical example is a chat client and a server. When a session is suspended information on every actor's data, e.g. user name, connections, type of application and information for child sessions should be stored.

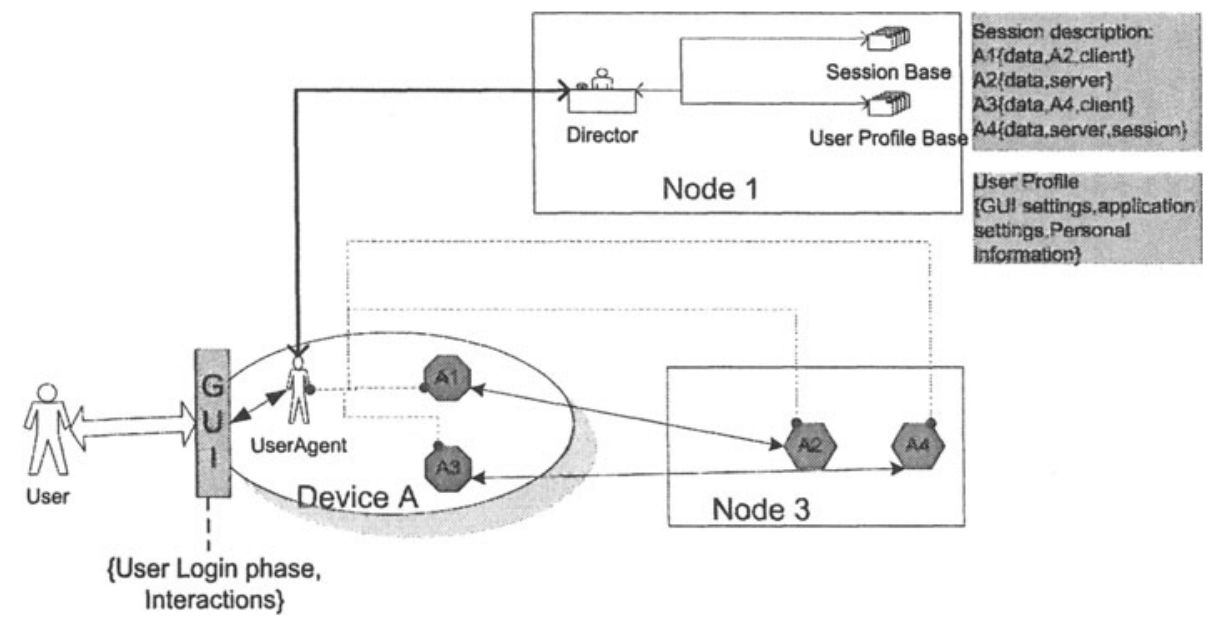

Figure 3. Session mobility: A user is interacting with the system through UserAgent and its session is stored in the director's Session Base.

User's Login phase is central to the definition of users identity, characterization of device capabilities, resumption of user sessions, and transfer of personal content. It is used by the director to provide users with proper access rights and profiles. Therefore, there are two types of logins in a multi domain environment, as shown in Figure 4. Eventually, users could 
access their home domain after some inter-director negotiation and authentication.

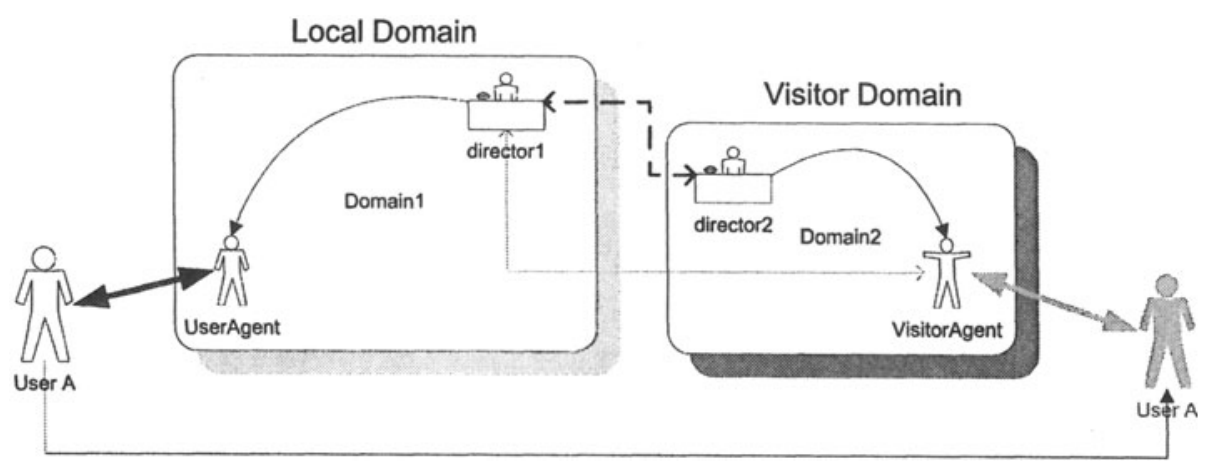

Figure 4. User mobility: User A is accessing his home domain from Domain2.

\section{EXPERIMENTATION}

The basic configuration that we are experimenting with is based on two subnets, within one LAN network, which have two wireless parts. These are referred to by LAN1 and LAN2 in Figure 5. Two sets of support systems and applications run at these subnets with two different directors. In this configuration domains are viewed as an IP address range controlled by a subnet mask, and therefore directors consider plugged in nodes by their IP addresses. A mobile node, laptop computer, is plugged in at these two domains and considered as a mobile device running and participating in the two applications. This mobile node acquires two different IP addresses before the user can login. Different Mobility scenarios are being performed, e.g. user login and logoff, session suspend and resume, visitor domain login, device identification, etc.

\section{CONCLUSION}

This paper demonstrates Personal Mobility approach in TAPAS. Basic user, device and session mobility issues are introduced. It provides a general view of requirements for supporting this kind of mobility, and some implementation and experimentation features are pointed at. Furthermore, a closer look is provided at Wireless Internet applications that could be modelled and developed using this platform. 


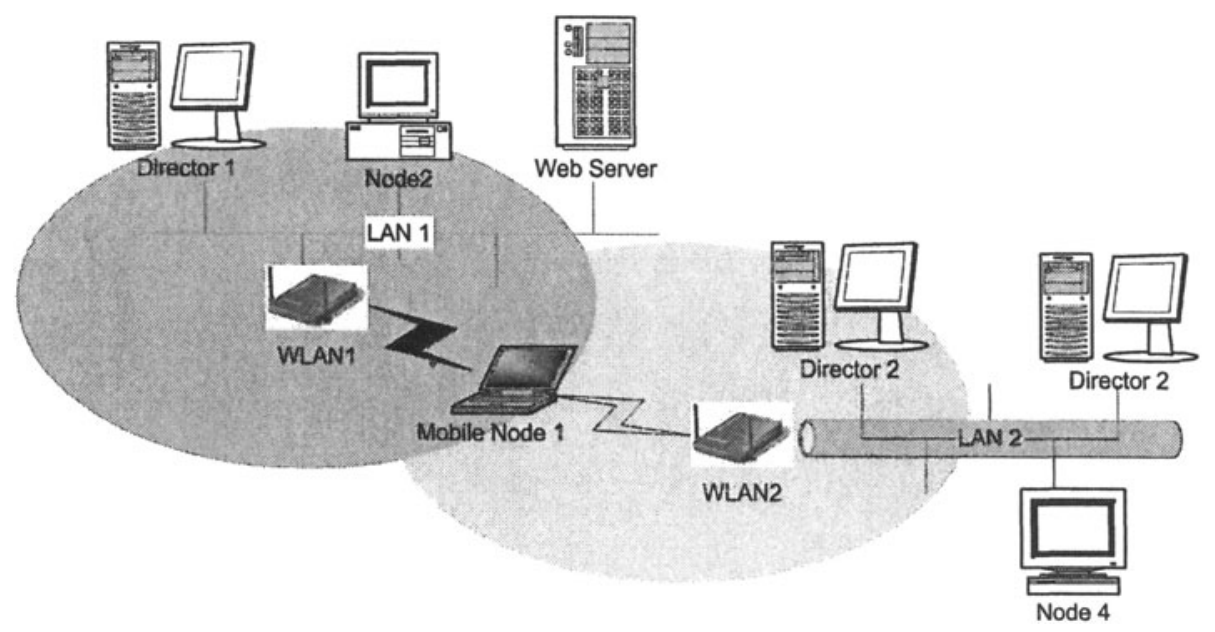

Figure 5. Experimentation with two domains and a mobile device.

Future work is meant to concentrate on two issues. First, extending the platform to handle the wireless aspects, such as: signal quality variation, connection recovery, and intelligence on missed nodes and partitioned networks. Second, providing support and basic functions for small devices, such as PDAs and Java phones.

\section{REFERENCES}

1. Aagesen, F. A., Helvik, B. E., uwongse, V., Meling, H., Bræk, R., Johansen, U. "Towards a plug and play architecture for telecommunications". Paper presented at the IFIP TC6 Fifth International Conference on Intelligence in Networks, Bankok, November 1999.

2. Mazen Malek Shiaa and Finn Arve Aagesen. "Mobility management in a Plug and Play Architecture", IFIP TC6 Seventh International Conference on Intelligence in Networks, Saariselka, Finland, April 2002. Available from:

http://www.item.ntnu.no/ plugandplay/publications/ 